\title{
Development of coherence in the high-gain Compton free-electron laser
}

\author{
C. Penman \\ Faculteit der Technische Natuurkunde, Universiteit Twente, Postbus 217, 7500 AE Enschede, Netherlands
}

Received 2 January 1992; revised manuscript received 30 March 1992

\begin{abstract}
The start-up of the free-electron laser is treated by linear analysis in the high-gain Compton regime. The results are compared with computer simulation.
\end{abstract}

\section{Introduction}

Theoretical treatments of the free-electron laser are based on the coupled Maxwell and Lorentz equations for the laser field and electron motion, for which the condition of zero field in a uniform mono-energetic beam forms an unstable trivial solution. The instability enables a real free-electron laser without injected signal to start itself from noise either in the field or in the electron beam. Computer simulations, on the other hand, usually proceed from a small arbitrary value of input field, since otherwise the instability would not show itself. The instability then develops in the usual exponential fashion (the growth of the field itself is not initially exponential because of a mixing of eigenmodes of the system, none of which is at first dominant). Such a numerical procedure is subject to several objections. The time or distance required to reach saturation will depend on the arbitrary initial value of the field and will thus give no indication of the value to be expected in reality; the procedure is clearly invalid unless the initial field is larger than the random fluctuations in the system which it is intended to model. Even if the level of these fluctuations is used as an initial value for the field, the time to saturation, which involves an exponential growth, will still be incorrectly predicted, since the initial evolution of the electron bunching and field is an incoherent process with linear growth; the transition from this stochastic process into a coherent one, with exponential growth, will not be even qualitatively reproduced. Studies with the low-gain Compton free-electron laser [1,2] show an initial phase of linear growth in the intensity which is succeeded by exponential growth until saturation is approached. This linear phase is due not to the modemixing mentioned earlier but to the incoherent addition of fields from an increasing number $N$ of different electrons, with a $\sqrt{N}$ dependence for the field amplitude. As bunching becomes significant and the growth in intensity exponential, the increasing coherence time of the field can be seen in a narrowing of the spectrum. The purpose of the present study is to extend this treatment to the high-gain Compton regime.

To get an understanding of the problem one must seek analytic solutions. The FEL equations are nonlinear and somewhat complicated. Fortunately, experience with the low-gain case indicates that the process of start-up takes place while the system is still in the linear regime. The main complication remaining, the different characteristics of the differential operators for electrons and field, can be dealt with by Fourier [2] or Laplace [3] transforms. It is important to note that the relative movement or slip between the electron and optical pulses can never be neglected in analysis of free-electron laser initiation, even under the condition of long pulses which suffices to justify such neglect in the subsequent evolution. This is because the theory is essentially concerned with an inhomogeneity in the internal timestructure of the pulses. 


\section{Model and analytic results}

With Bonifacio's normalization [3] but with a slightly different coordinate system in which $\tau$ represents the electron-beam local time, the Compton FEL equations for the electron phase variables $\theta_{j}$ and normalized radiation vector potential $a$ are

$\partial^{2} \theta_{j} / \partial Z^{2}=-\left[a \exp \left(\mathrm{i} \theta_{j}\right)+\right.$ c.c. $]$,

$\left(\frac{\partial}{\partial Z}-\frac{1}{V} \frac{\partial}{\partial \tau}\right) a=\langle\exp (-\mathrm{i} \theta)\rangle$,

where $V \equiv \beta_{z} /\left(1-\beta_{z}\right)$ gives the rate of relative movement or slip between optical and electron pulses in terms of the average initial axial electron speed $c \beta_{z}$ inside the undulator, and other symbols are defined in table 1. As in ref. [2], the procedure will be to separate the average $\langle\exp (-i \theta)\rangle$ into a coherent part $\langle\exp (-\mathrm{i} \theta)\rangle_{c}$, consisting of departures from even electron-spacing due to the ponderomotive potential and evolving with $Z$, and part $f(\tau)$ representing the contribution of stochastic uneven spacing which remains approximately constant and depends only on the position $\tau$ within the electron pulse. The statistical properties of $f$ are defined by ensemble-averages: $f$ is considered to have different forms for each realization in the ensemble, but the ensemble average $f$ satisfies

$\overline{f(\tau)}=0, \overline{f(\tau) f^{*}\left(\tau^{\prime}\right)}=\delta\left(\tau-\tau^{\prime}\right)$,

for all $\tau$ and $\tau^{\prime}$. With this separation, eq. (2) becomes

$$
\left(\frac{\partial}{\partial Z}-\frac{1}{V} \frac{\partial}{\partial \tau}\right) a=\langle\exp (-\mathrm{i} \theta)\rangle_{\mathrm{c}}+g f(\tau) \text {. }
$$

The constant $g$ is determined by requiring the energy contribution of an interval $\tau$ of a noisy electron pulse of density $n_{\mathrm{e}}$ and cross-sectional area $\sigma$ to be $n_{\mathrm{e}} \sigma \tau_{\mathrm{w}} /$ $\left(2 k_{\mathrm{w}} \rho\right)$ times that from a single electron, both after the same propagation distance $Z$. (In the case of a single electron the evaluation of the delta-function in the electron-density is most easily performed in terms of the original coordinates $z$ and $t$, and the duration of the emission is equal to the slip time $z /(c V)$.) The result thus obtained is that $g^{-1}$ is the square root of the number of electrons in unit lengths of beam, or

$g^{2}=2 p k_{\mathrm{w}} /\left(\sigma n_{\mathrm{e}}\right)$.

In integrating eqs. (1) and (4) a small field and small fractional energy change will again be assumed as in ref. [2], but the assumption there of small fractional change in the field will no longer be made, so allow-

Table 1

\begin{tabular}{ll}
\hline Meanings of symbols & \\
\hline $\begin{array}{l}a_{\mathrm{r}}=e A_{\mathrm{r}} / m c \\
a_{\mathrm{w}}=e A_{\mathrm{w}} / m c\end{array}$ & $\begin{array}{l}\text { normalized radiation vector potential } \\
k_{\mathrm{w}}\end{array}$ \\
$\gamma$ & $\begin{array}{l}\text { normalized undulator vector potential } \\
\text { undulator wavenumber } \\
\text { average initial electron energy divided by } m c^{2}\end{array}$ \\
$\beta_{z}=\left(1+\frac{1+a_{\mathrm{w}}^{2}}{2 \gamma^{2}}\right)^{-1}$ & average initial electron axial speed divided by $c$ \\
$n_{\mathrm{c}}$ & electron density \\
$\omega_{\mathrm{p}}=e^{2} n_{\mathrm{e}} / \epsilon_{0} m_{0}$ & plasma frequency \\
$\rho=\frac{1}{\gamma}\left(\frac{a_{\mathrm{w}}}{4} \frac{\omega_{\mathrm{p}}}{c k_{\mathrm{w}}}\right)^{2 / 3}$ & FEL parameter \\
$Z=2 k_{\mathrm{w}} \rho z$ & normalized axial coordinate \\
$\tau=2 k_{\mathrm{w}} \rho\left(c t-z / \beta_{\mathrm{z}}\right)$ & normalized electron local time \\
$V=\beta_{z} /\left(1-\beta_{z}\right)$ & slip factor \\
$\theta_{j}$ & ponderomotive phase coordinate, $j$ th electron \\
$a(z, \tau)=\frac{\omega}{\omega_{\mathrm{p}} \sqrt{\rho \gamma}}$ & radiation vector potential, Bonifacio's normalization \\
$A(Z, \omega)$ & Fourier transform of $a(Z, \tau)$ with respect to $\tau$ \\
$\nu$ & detuning
\end{tabular}


ing for high gain. The model differs from ref. [3] in the separation of bunching terms (4) and the statistical treatment of the second term.

Integrating eq. (1) twice, assuming small field $a$, averaging and taking the Fourier transform $A(Z, \omega)$ of $a(Z, \tau)$ and $F(\omega)$ of $f(\tau)$ gives

$\frac{\partial A}{\partial Z}+\mathrm{i} \nu A=\left(\mathrm{i} \int_{0}^{z}\left(Z-Z^{\prime}\right) A\left(Z^{\prime}, \omega\right) \mathrm{d} Z^{\prime}\right)-g F(\omega)$,

where $|\nu|=|\omega| / V$, with sign chosen opposite to the detuning of Bonifacio [4] to suit later presentation of results as spectra plotted against $\nu$. Differentiating eq. (6) twice then gives

$\frac{\partial^{3} A}{\partial Z^{3}}+\mathrm{i} \nu \frac{\partial^{2} A}{\partial Z^{2}}=\mathrm{i} A(Z, \omega)$.

Let

$A(Z, \omega)=\sum C_{k} \exp \left(\mathfrak{i}_{k} Z\right)$,

where $C_{k}(k=0,1,2)$ are determined by the boundary conditions

$A(0, \omega)=A_{0}(\omega)$,

$\partial A(0, \omega) / \partial Z=-\mathrm{i} \nu A_{0}-g F(\omega)$,

$\partial^{2} A(0, \omega) / \partial Z^{2}=-\nu^{2} A_{0}+\mathrm{i} \nu g F(\omega)$.

By eqs. (7) and (8), $\lambda_{k}(k=0,1,2)$ satisfy

$\lambda^{3}+\nu \lambda^{2}+1=0$.

Every function of $Z$ which satisfies eq. (7) with boundary conditions sufficient to determine $C_{k}$ $(k=0,1,2)$ has the form of eq. (8); the only other functions which could satisfy eq. (6) would differ from this form by terms respectively linear and quadratic in $Z$, but from the form of eq. (6) it follows that the coefficients of these terms are both zero. The effect of the incoherent term $-g F$ in eq. (6) appears only as a boundary condition for eq. (7).

The discriminant $D$ of eq. (10) is given by

$D^{2}=-(\nu / 3)^{6}+r^{2}, \quad r=-\frac{1}{4}-(\nu / 3)^{3}$,

and the roots are therefore

$\lambda=s+d-\nu / 3$,

$\lambda_{1}=-\frac{1}{2}(s+d)+\mathrm{i}(\sqrt{3} / 2)(s-d)-\nu / 3$,

$\lambda_{2}=-\frac{1}{2}(s+d)-\mathrm{i}(\sqrt{3} / 2)(s-d)-\nu / 3$, where

$s^{3} \equiv r+D, \quad d^{3} \equiv r-D$.

The ultimate growth rate, given by the dominant eigenvalue, is $\sqrt{3} / 2$ in the case of zero detuning $(\nu=0)$ and

$(\sqrt{3} / 2)\left[1+2(\nu / 3)^{3}\right]^{1 / 3} \approx(\sqrt{3} / 2)\left[1+\frac{2}{3}(\nu / 3)^{3}\right]$,

in the limit of large current (because of the scaling of $Z$ and $\tau$ with the cube root of the current in Bonifacio's normalization [4], this limit implies $\left.\nu^{3} \ll 1\right)$.

By eq. (9), $C_{k}(k=0,1,2)$ are the solution of the linear system

$M C=b$,

where

$m_{i j}=\lambda_{j}^{i}, \quad i, j=0,1,2$,

and

$\boldsymbol{b}=\left[A_{0}(\omega),-\nu A_{0}+\mathrm{i} g F(\omega), \nu^{2} A_{0}-\mathrm{i} \nu g F(\omega)\right]^{\mathrm{T}}$,

(array subscripts in this work begin at 0 ). Since the determinant is

$|M|=-\mathrm{i} 2 \times 3^{3 / 2}\left[\frac{1}{4}+(\nu / 3)^{3}\right]^{1 / 2}$,

the solution exists except at $\nu=-3 / 2$, the point of coincident roots of the cubic, where a limit can be taken. For a general set of boundary conditions $b$ the solution is

$C_{0}=\frac{\lambda_{1} \lambda_{2} b_{0}-\left(\lambda_{1}+\lambda_{2}\right) b_{1}+b_{2}}{\left(\lambda_{0}-\lambda_{1}\right)\left(\lambda_{0}-\lambda_{2}\right)}$,

with similar expressions (under cyclic permutation of subscripts) for $C_{1}$ and $C_{2}$.

In contrast to the incoherent term, the first term on the right of eq. (6) affects only the cubic and not the boundary conditions. In its absence, $\lambda_{0}=-\nu$, $\lambda_{1}=\lambda_{2}=0$,

$C_{0}=A_{0}-(\mathrm{i} / \nu) g F(\omega)$,

and by substituting eq. (8) in eq. (6) one finds the constant term in $A(Z, \omega)$ :

$C_{1}+C_{2}=(\mathrm{i} / \nu) g F(\omega)$.

Since the terms on the right of eq. (6) are associated respectively with stimulated and spontaneous emission, the spontaneous spectrum $I_{\text {spon }}(Z, \nu)$ is deter- 
mined from the solution with no first term

$$
\begin{aligned}
& A(Z, \omega)=A_{0}(\omega) \exp (-\mathrm{i} \nu Z) \\
& \quad+(\mathrm{i} / \nu) g F(\omega)[1-\exp (-\mathrm{i} \nu Z)],
\end{aligned}
$$

by the identity

$$
\begin{aligned}
& \overline{A(Z, \omega) A^{*}\left(Z, \omega^{\prime}\right)} \\
& \quad=\sqrt{2 \pi} I_{\text {spon }}(Z, \omega / V) \delta\left(\omega-\omega^{\prime}\right) .
\end{aligned}
$$

If $A_{0}(\omega)=0$, this gives

$I_{\text {spon }}(Z, \nu)=\left(g^{2} / \sqrt{2 \pi}\right)(2 / \nu)^{2} \sin ^{2}(\nu Z / 2)$,

by the correlation, reciprocal to eq. (3), of $F$. This has a peak (at $\nu=0$ ) of

$I_{\text {spon,max }}(Z)=\left(g^{2} / \sqrt{2 \pi}\right) Z^{2}$,

and a width (approximate half-width at half maximum) of $\pi / Z$.

Proceeding now to solutions with particular boundary conditions $b$, one finds, first, for the case of input field but no noise

$\boldsymbol{b}=A_{0}\left(1,-\nu, \nu^{2}\right)^{\mathrm{T}}, \quad A_{0}(\omega)=A(\omega, 0)$,

$C_{0}=A_{0} \frac{\lambda_{1} \lambda_{2}+\left(\lambda_{1}+\lambda_{2}\right) \nu+\nu^{2}}{\left(\lambda_{0}-\lambda_{1}\right)\left(\lambda_{0}-\lambda_{2}\right)}$,

$C_{1}=A_{0} \frac{\lambda_{2} \lambda_{0}+\left(\lambda_{2}+\lambda_{0}\right) \nu+\nu^{2}}{\left(\lambda_{1}-\lambda_{2}\right)\left(\lambda_{1}-\lambda_{0}\right)}$

$C_{2}=A_{0} \frac{\lambda_{0} \lambda_{1}+\left(\lambda_{0}+\lambda_{1}\right) \nu+\nu^{2}}{\left(\lambda_{2}-\lambda_{0}\right)\left(\lambda_{2}-\lambda_{1}\right)}$.

That is,

$$
\begin{aligned}
C_{0} & =\left(A_{0} /|M|\right)\left(\lambda_{1}-\lambda_{2}\right)\left[\lambda_{1} \lambda_{2}+\left(\lambda_{1}+\lambda_{2}\right) \nu+\nu^{2}\right] \\
& =\left(A_{0} /|M|\right) \mathrm{i} \sqrt{3}(s-d)\left[s^{2}+d^{2}-s d\right. \\
& \left.-(s+d)(2 \nu / 3)+(2 \nu / 3)^{2}\right],
\end{aligned}
$$

with similar equations, involving phase-factors $\exp [i( \pm 2 / 3) \pi]$ multiplying $s$ and $d$, for $C_{1}$ and $C_{2}$. With these, eq. (8) gives the evolution in the linear regime as calculated, e.g., by Bonifacio [3].

The opposite case of noise but no input field is the one of interest for this work. Here

$$
\begin{aligned}
& \boldsymbol{b}=g F(\omega)(0, \mathrm{i},-\mathrm{i} \nu)^{\mathrm{T}} \\
& C_{0}=-\mathrm{i} g F \frac{\lambda_{1}+\lambda_{2}+\nu}{\left(\lambda_{0}-\lambda_{1}\right)\left(\lambda_{0}-\lambda_{2}\right)}=\mathrm{i} \frac{g F}{|M|} \lambda_{0}\left(\lambda_{1}-\lambda_{2}\right),
\end{aligned}
$$

$$
\begin{aligned}
& C_{1}=\mathrm{i}(g F /|M|) \lambda_{1}\left(\lambda_{2}-\lambda_{0}\right), \\
& C_{2}=\mathrm{i}(g F /|M|) \lambda_{2}\left(\lambda_{0}-\lambda_{1}\right) .
\end{aligned}
$$

The ratio of the spectrum to the spontaneous peak, eq. (15), is

$$
\begin{aligned}
& \frac{I(Z, \nu)}{I_{\text {spon,max }}(Z)}=-\frac{\sqrt{2 \pi}}{|M|^{2} Z^{2}} \\
& \times\left|\sum_{k=0}^{2} \lambda_{k}\left(\lambda_{k+1}-\lambda_{k+2}\right) \exp \left(\mathrm{i} \lambda_{k} Z\right)\right|^{2},
\end{aligned}
$$

(all subscripts being taken modulo 3 ), which is plotted against $\nu$ for various values of $Z$ in fig. 1 . The main lobe of the spectrum increases in height with $Z$, and also gets narrower; in contrast to the low-gain case [2], the spectral peak does not evolve towards the turning-point of the spontaneous spectrum ( $\nu \simeq 2.606$ ), but moves a small distance from $\nu=0$ (because of the mode mixing mentioned above) and then gradually back again. Figure 2 shows the diminution of the spectral width $\Delta \nu$ with propagating distance $Z$ indicating a progressive increase in coherence length $\sim 1 /(V \Delta \nu)$. The cumulative slip distance $Z / V$ forms a theoretical upper bound for the coherence length [6]; in the present model the reciprocals of the first few spectral width values are exactly proportional to propagation distance, with constant of order unity, showing that, to within an order of magnitude, this theoretical upper bound is actually attained in the initial stages.

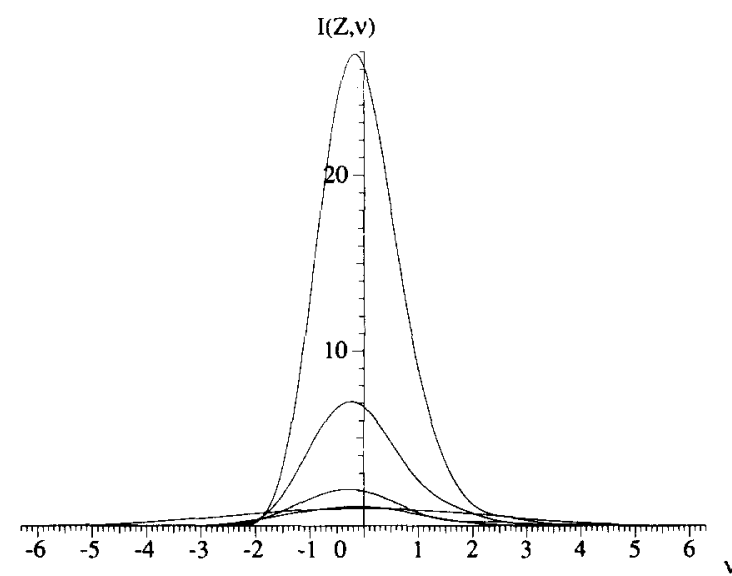

Fig. 1. Analytic evolution of spectra. Normalized propagation distance $Z=1,2,3,4,5$ (reading upwards). 


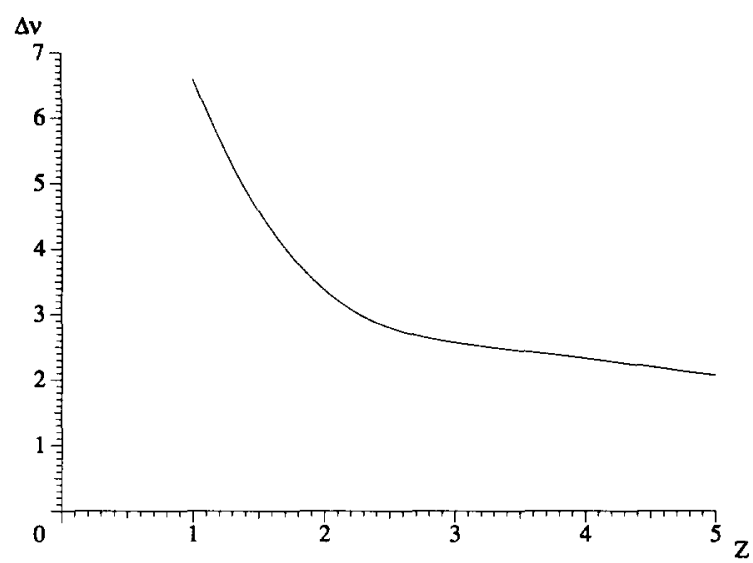

Fig. 2. Diminution of spectral width with propagation distance, demonstrating development of coherence.

\section{Numerical simulation}

To obtain correct results from a numerical integration of the free-electron laser equations, a statistical treatment of the noise in the electron phase-distribution is needed, so that the noise level in a simulation can be corrected for the unrealistically small number of electron phase-values necessarily used on a computer. The method, based on a comparison of statistics in the real and the numerical situations, is described in a separate publication [5]; it has been shown to give agreement with low-gain start-up theory [2].

In figs. 3 to 8 the results of a numerical integration of the Compton free-electron laser equations (1) and (2), using the correction for the electron-noise statistics described in ref. [5], are shown. These represent an instance or realization of the process over which ensemble-averages were taken in the theory. The time-profiles (odd-numbered figures) show the relative movement of electron and optical pulses which is an essential part of any start-up theory. The optical intensity pulse, shown as a function of its own local time $2 \rho k_{\mathrm{w}}(c t-z)$, spreads to the right or trailing side as $Z$ increases, reflecting the fact that the electron pulse gets slightly left behind. To avoid broadening the spectrum a pulse much longer than the reciprocal of the expected bandwidth, and therefore much longer than the slip time, has been used. This has the consequence that, in contrast to the lowgain case, the particular stochastic features of the re-

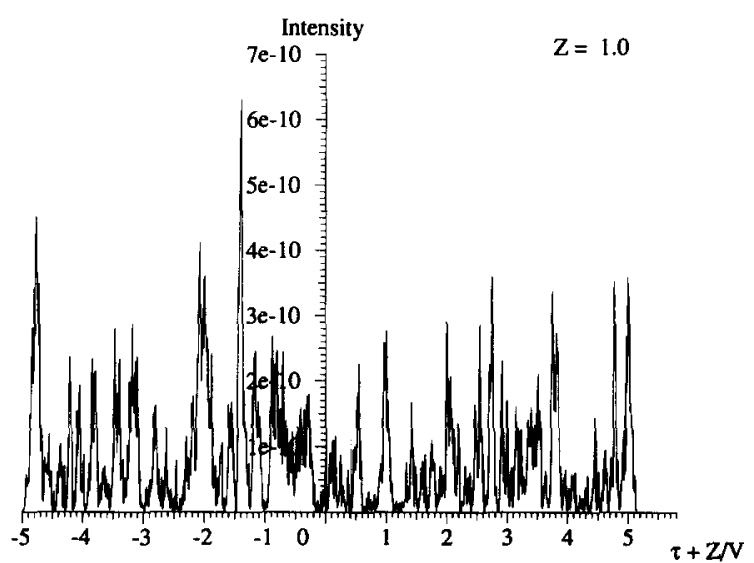

Fig. 3. Numerical simulation. FEL parameter $\rho=0.01$, normalized propagation distance $Z=1$. Time profile of field.

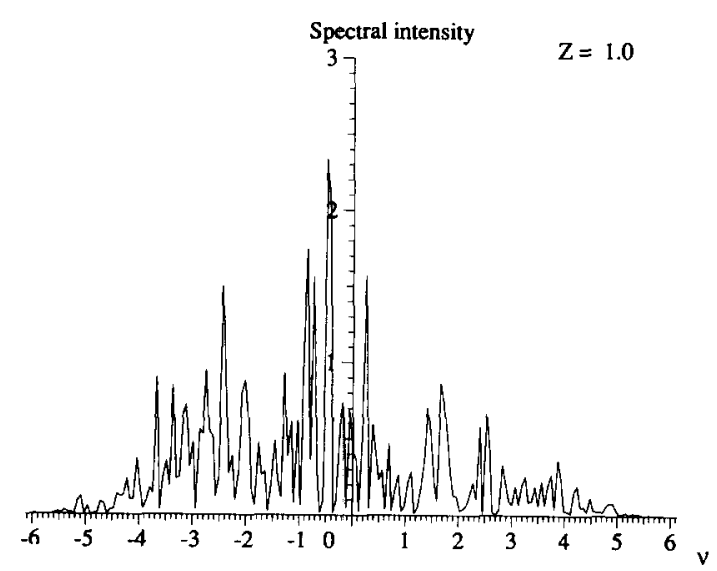

Fig. 4. Numerical simulation. FEL parameter $\rho=0.01$, normalized propagation distance $Z=1$. Spectrum of field.

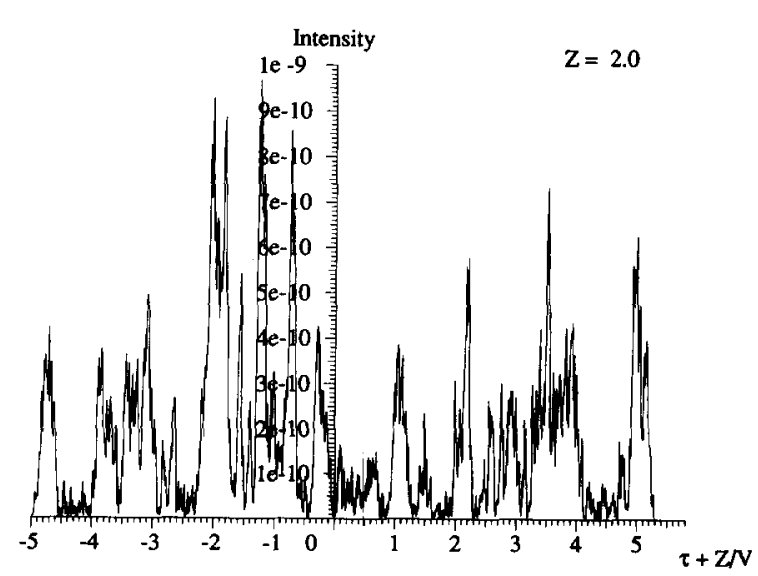

Fig. 5. $\rho=0.01, Z=2$. Time profile. 


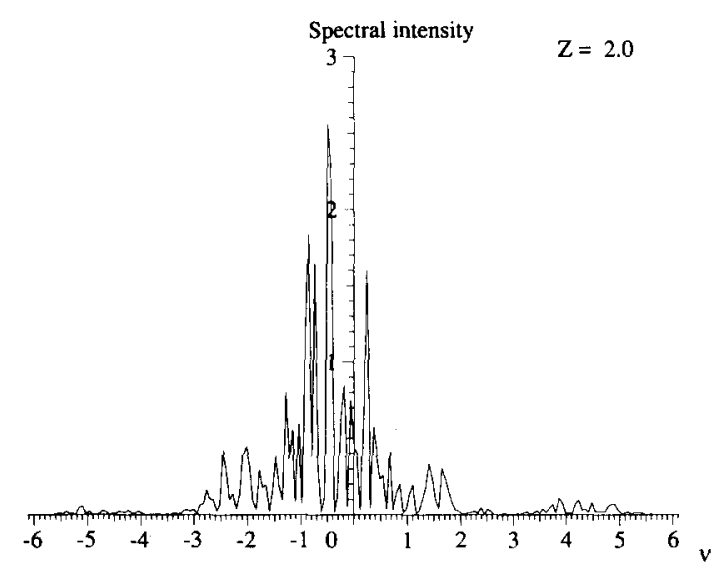

Fig. 6. $\rho=0.01, Z=2$. Spectrum.

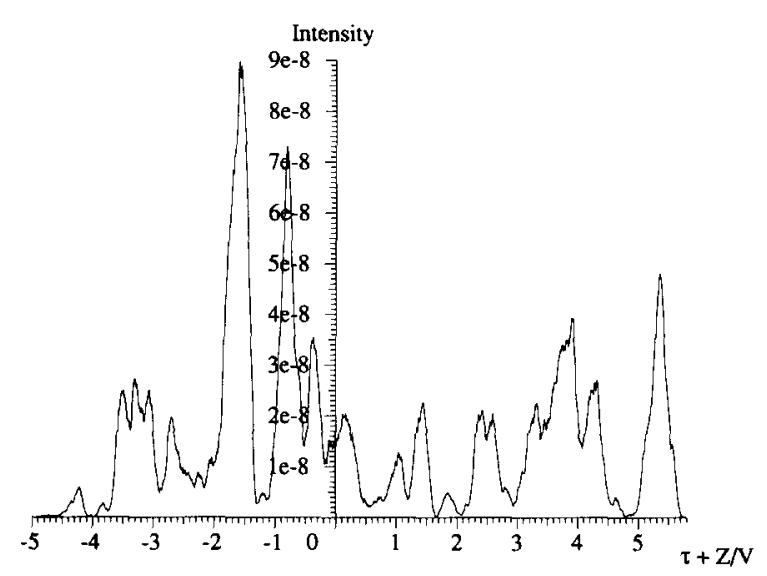

Fig. 7. $\rho=0.01, Z=5$. Time profile.

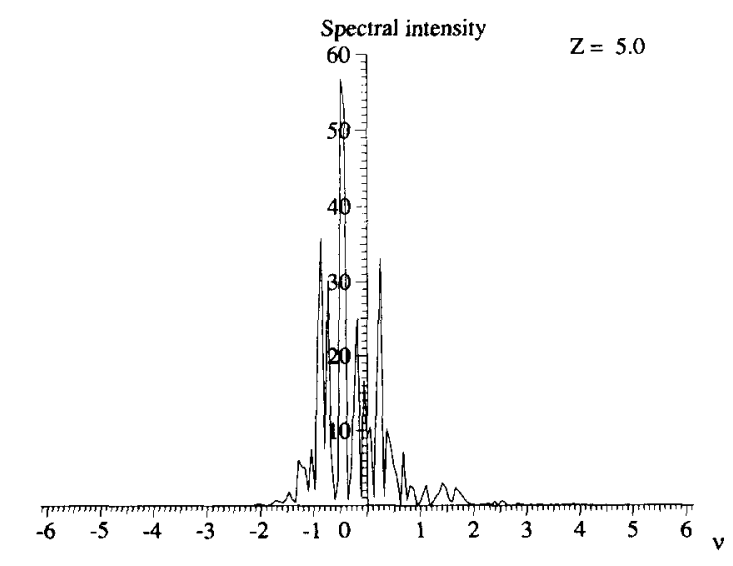

Fig. 8. $\rho=0.01, Z=5$. Spectrum. alization remain conspicuous even when the spectrum has considerably narrowed (fig. 8). The reason is that, because of the high gain, these features get magnified before they have been much spread out by the slippage (by contrast, pulses which are not longer than the slip time rapidly become smooth over their whole width). In spite of the randomness which is therefore present, the qualitative behaviour of fig. 1 is evident in the spectra (even-numbered figures).

\section{Conclusion}

The evolution of the radiation spectrum of the freeelectron laser during start-up can be predicted analytically in the ensemble average. In the presence of high gain, the individual instances of the process differ from the average much more than with low gain. This may explain apparently random features observed in the output of pulses from high-gain experiments.

\section{Acknowledgements}

This work was funded by the Foundation for Fundamental Research on Matter, Utrecht, the Netherlands. I have benefited from helpful conversations with Peter van der Slot.

\section{References}

[1] P. Sprangle, C.M. Tang and I. Bernstein, Phys, Rev. A 28 (1983) 2300.

[2] J.N. Elgin and C. Penman, Phys. Lett. 114 A (1986) 227.

[3] R. Bonifacio, C. Maroli and N. Piovella, Optics Comm. 68 (1988) 369.

[4] R. Bonifacio, F. Casagrande, G. Gerchioni, L. de Salvo Souza and P. Pierini, in: High gain, high power free-electron laser: physics and application to $\mathrm{TeV}$ particle acceleration, eds. $\mathrm{R}$. Bonifacio, L. de Salvo Souza and C. Pellegrini (NorthHolland, Amsterdam, 1989).

[5] C. Penman and B.W.J. McNeil, Optics Comm. 90 (1992) 82.

[6] E.R. Stanford and T.M. Antonsen Jr., Nucl. Instrum. Methods, Phys. Res. A 304 (1991) 659. 